Bull. Korean Math. Soc. 49 (2012), No. 1, pp. 57-61

http://dx.doi.org/10.4134/BKMS.2012.49.1.057

\title{
DISCRETENESS BY USE OF A TEST MAP
}

\author{
LiUlan Li AND Xi Fu
}

\begin{abstract}
It is well known that one could use a fixed loxodromic or parabolic element of a non-elementary group $G \subset M\left(\overline{\mathbb{R}}^{n}\right)$ as a test map to test the discreteness of $G$. In this paper, we show that a test map need not be in $G$. We also construct an example to show that the similar result using an elliptic element as a test map does not hold.
\end{abstract}

\section{Introduction}

The discreteness has been a fundamental and important problem in $M\left(\overline{\mathbb{R}}^{n}\right)$ since Jørgensen [2] established the following well-known discreteness criterion in 1976.

Theorem $J$ ([2, Proposition 2]). A non-elementary subgroup $G$ of $M\left(\overline{\mathbb{R}}^{2}\right)$ is discrete if and only if each two-generator subgroup of $G$ is discrete.

In 2004, Yueping Jiang [1] generalized Theorem $J$ and proved the following.

Theorem $Y$ ([1, Theorem 2]). Let $G \subset M\left(\overline{\mathbb{R}}^{n}\right)$ be non-elementary. Then $G$ is discrete if and only if $W(G)$ is discrete and each two-generator non-elementary subgroup is discrete.

Here $W(G)=\{f \in G: f$ fixes every limit point of $G\}$.

Jiang also gave an example to show that the condition that " $W(G)$ is discrete" is necessary when $n \geq 3$.

In [8], Xiantao Wang et al generalized Theorem $J$ by using loxodromic or parabolic elements as follows.

Theorem $W_{1}$ ([8, Theorem 3.1]). Let $G \subset M\left(\overline{\mathbb{R}}^{n}\right)$ be non-elementary. Then $G$ is discrete if and only if $W Y(G)$ is discrete and each non-elementary subgroup generated by two elements of $G_{f}$ is discrete, where $f \in G$ is loxodromic.

Received January 11, 2010; Revised May 18, 2010.

2010 Mathematics Subject Classification. Primary 30F40; Secondary $20 \mathrm{H} 10$.

Key words and phrases. discreteness, non-elementary Möbius group, test map.

The research was supported by the Science and Technology Development Program of Hengyang (No. 2010KJ22), NSF of Hunan (No. 10JJ4005) and Hunan Provincial Education Department (No. Q12034). 
Theorem $W_{2}\left(\left[8\right.\right.$, Theorem 3.2]). Let $G \subset M\left(\overline{\mathbb{R}}^{n}\right)$ be non-elementary containing parabolic elements. Then $G$ is discrete if and only if $W Y(G)$ is discrete and each non-elementary subgroup generated by two elements of $G_{f}$ is discrete, where $f \in G$ is parabolic.

Here $G_{f}, W Y(G)$ in Theorems $W_{1}$ and $W_{2}$ are defined as follows:

$G_{f}=\{g \in G: g$ is conjugate to $f$ and $\langle f, g\rangle$ is non-elementary $\} \cup\{f\}$,

$W Y(G)=\{g \in G: g$ fixes every fixed point of each loxodromic element of $\mathrm{G}\}$.

Obviously, if $G$ is non-elementary, then $W(G)=W Y(G)$.

The novelty of Theorems $W_{1}$ and $W_{2}$ is that the discreteness of $G$ is totally determined by a loxodromic (resp. parabolic) element of $G$.

See $[3] \sim[7],[4] \sim[14]$ for the generalization of Theorem $J$ and other discussions in this line.

In this paper, we first make further generalizations of Theorems $W_{1}$ and $W_{2}$ and obtain

Theorem 1.1. Let $G \subset M\left(\overline{\mathbb{R}}^{n}\right)$ be a non-elementary group and $f \in M\left(\overline{\mathbb{R}}^{n}\right)$ loxodromic. If $W Y(G)$ is discrete and each non-elementary group $\left\langle f, g f g^{-1}\right\rangle$ is discrete, where $g \in G$, then $G$ is discrete.

Theorem 1.2. Let $G \subset M\left(\overline{\mathbb{R}}^{n}\right)$ be a non-elementary group and $f \in M\left(\overline{\mathbb{R}}^{n}\right)$ parabolic. If $W Y(G)$ is discrete and each non-elementary group $\left\langle f, g f g^{-1}\right\rangle$ is discrete, where $g \in G$, then $G$ is discrete.

Remark 1.3. Theorems 1.1 and 1.2 are generalizations of Theorems $W_{1}$ and $W_{2}$, which show that the test map need not be in $G$.

Naturally, we may ask the following problem:

Conjecture 1.4. Let $G \subset M\left(\overline{\mathbb{R}}^{n}\right)$ be a non-elementary group and $f \in M\left(\overline{\mathbb{R}}^{n}\right)$ elliptic. If $W Y(G)$ is discrete, and each non-elementary group $\left\langle f, g f g^{-1}\right\rangle$ is discrete, where $g \in G$, then $G$ is discrete.

We construct an example to show that Conjecture 1.4 does not hold. The example and the proofs of Theorems 1.1 and 1.2 will be presented in Section 3 .

\section{Preliminaries}

For $n \geq 2$, we denote by $\overline{\mathbb{R}}^{n}$ the one-point compactification of $\mathbb{R}^{n}$ obtained by adding $\infty$. The group of orientation-preserving Möbius transformations of $\overline{\mathbb{R}}^{n}$ is denoted by $M\left(\overline{\mathbb{R}}^{n}\right)$. We regard $\overline{\mathbb{R}}^{n}$ as the boundary at infinity of the hyperbolic $(n+1)$-space $\mathbb{H}^{n+1}$ and let $\overline{\mathbb{H}}^{n+1}=\mathbb{H}^{n+1} \cup \overline{\mathbb{R}}^{n}$. Any Möbius transformation of $\overline{\mathbb{R}}^{n}$ is extended to an isometry of $\mathbb{H}^{n+1}$.

For a non-trivial element $g \in M\left(\overline{\mathbb{R}}^{n}\right)$, we let

$$
f i x(g)=\left\{x \in \overline{\mathbb{H}}^{n+1}: g(x)=x\right\} \text {. }
$$


$g$ is called loxodromic if it has two fixed points in $\overline{\mathbb{R}}^{n}$ and none in $\mathbb{H}^{n+1}$, parabolic if it has only one fixed point in $\overline{\mathbb{R}}^{n}$ and none in $\mathbb{H}^{n+1}$, and elliptic if it has a fixed point in $\mathbb{H}^{n+1}$.

Let $G \subset M\left(\overline{\mathbb{R}}^{n}\right)$ be a group. For a point $x \in \overline{\mathbb{H}}^{n+1}$, the set $G(x)=\{g(x)$ : $g \in G\}$ is called $G$-orbit of $x$. We call $G$ elementary if $G$ has a finite $G$ orbit. Otherwise, we say that $G$ is non-elementary. The limit set $L(G)$ of a non-elementary group $G \subset M\left(\overline{\mathbb{R}}^{n}\right)$ is defined as follows:

$$
L(G)=\overline{G(z)} \cap \overline{\mathbb{R}}^{n}
$$

for some $z \in \mathbb{H}^{n+1}$, where the overline denotes the closure in $\mathbb{H}^{n+1} \cup \overline{\mathbb{R}}^{n}$ and $G(z)$ the $G$-orbit of $z$. We call points of $L(G)$ limit points.

If there exists a sequence of distinct elements of $G$ converging to the identity, then we say that $G$ is not discrete. Otherwise, we say that $G$ is discrete.

For $g_{r}=\left(\begin{array}{ll}a_{r} & b_{r} \\ c_{r} & d_{r}\end{array}\right) \in M\left(\overline{\mathbb{R}}^{n}\right)(r=1,2)$, we define

$$
\left\|g_{1}-g_{2}\right\|=\left(\left|a_{1}-a_{2}\right|^{2}+\left|b_{1}-b_{2}\right|^{2}+\left|c_{1}-c_{2}\right|^{2}+\left|d_{1}-d_{2}\right|^{2}\right)^{\frac{1}{2}} .
$$

The following lemma is crucial for our investigation.

Lemma 2.1 ([13, Theorem 11]). Let $f, g \in M\left(\overline{\mathbb{R}}^{n}\right)$. If $\langle f, g\rangle$ is a discrete and non-elementary group, then

$$
\|f-I\| \cdot\|g-I\| \geq \frac{1}{36} .
$$

\section{Proofs of Theorems 1.1 and 1.2 and an example}

It suffices to prove Theorem 1.1 since the proof of Theorem 1.2 is similar.

Proof of Theorem 1.1. Since $G$ is non-elementary, under conjugation, we let $\sigma(L(G))$ be the minimal subspace of $\overline{\mathbb{R}}^{n}$ containing $L(G)$ (cf. [7]), and let $h_{1}$, $h_{2}, \ldots, h_{k} \in G$ be loxodromic such that no two of them have any fixed point in common and the set $\left\{f i x\left(h_{1}\right), f i x\left(h_{2}\right), \ldots, f i x\left(h_{k}\right)\right\}$ spans $\sigma(L(G))$.

Suppose, on the contrary, that $G$ is not discrete. Then there exists a sequence $\left\{g_{i}\right\} \subset G$ of distinct elements converging to the identity as $i \rightarrow \infty$. Since $W Y(G)$ is finite, by choosing a subsequence of $\left\{g_{i}\right\}$ (still denoted by $\left\{g_{i}\right\}$ ), if necessary, we may assume that there exists some $h_{j}$, say $h_{1}$, such that $f i x\left(h_{1}\right)$ is not contained in $f i x\left(g_{i}\right)$ for all $i$. Let $f i x\left(h_{1}\right)=\{a, b\}$, where $a$ is the attractive fixed point of $h_{1}$. We assume further that $a$ is not fixed by each $g_{i}$. We choose a neighborhood $U$ of $a$ such that $b \notin U$ and $U \cap f i x\left(h_{j}\right)=\emptyset$ for $j=2,3, \ldots, k$. Then there exists an integer $r$ such that $f i x\left(h_{1}^{r} g_{i} h_{1}^{-r}\right) \subset U \cup\{b\}$ for each $i$. We also choose an integer $u$ such that $U \cap f i x\left(h_{2}^{u} h_{1}^{r} g_{i} h_{1}^{-r} h_{2}^{-u}\right)=\emptyset$ for each $i$. We now still denote each $h_{2}^{u} h_{1}^{r} g_{i} h_{1}^{-r} h_{2}^{-u}$ by $g_{i}$. Then we get a sequence $\left\{g_{i}\right\} \subset G$ of distinct elements converging to the identity as $i \rightarrow \infty$ with $U \cap f i x\left(g_{i}\right)=\emptyset$ for each $i$.

If $b \notin f i x(f)$, then there exists an integer $s$ such that $f i x\left(h_{1}^{s} f h_{1}^{-s}\right) \subset U$. We let $p=h_{1}^{s}$ for this case. 
If $b \in f i x(f)$, then there exists a loxodromic element $h \in G$ such that

$$
f i x(h) \cap f i x\left(h_{1}\right)=\emptyset \text { and } f i x(h) \cap f i x(f)=\emptyset .
$$

Therefore, there exist integers $t, m$ such that $f i x\left(h_{1}^{m} h^{t} f h^{-t} h_{1}^{-m}\right) \subset U$. We let $p=h_{1}^{m} h^{t}$. So in either case, we find an element $p \in G$ such that $f i x\left(p f p^{-1}\right) \subset$ $U$.

Since

$$
\left\langle p f p^{-1}, g_{i} p f p^{-1} g_{i}^{-1}\right\rangle=p\left\langle f, p^{-1} g_{i} p f p^{-1} g_{i}^{-1} p\right\rangle p^{-1}
$$

and

$$
\left\langle f, p^{-1} g_{i} p f p^{-1} g_{i}^{-1} p\right\rangle=\left\langle f, p^{-1} g_{i} p f p^{-1} g_{i}^{-1} p f^{-1}\right\rangle,
$$

by the assumption and Lemma 2.1 , we know that $\left\langle p f p^{-1}, g_{i} p f p^{-1} g_{i}^{-1}\right\rangle$ is elementary for large enough $i$. Since $f$ is loxodromic and $g_{i} \rightarrow I$, we know

$$
f i x\left(g_{i}\right) \cap f i x\left(p f p^{-1}\right) \neq \emptyset
$$

for large enough $i$, which is a contradiction.

Example 3.1. Let $G_{0} \subset M\left(\overline{\mathbb{R}}^{2}\right)$ be a non-elementary and non-discrete group containing no elliptic elements, and let $G$ be the Poincaré extension of $G_{0}$ in $\overline{\mathbb{R}}^{4}$. Let $f$ be a rotation around $\overline{\mathbb{R}}^{2}$ of order $p(p \geq 3)$. Then $f$ is an elliptic element acting on $\overline{\mathbb{R}}^{4}$ whose fixed point set is $\overline{\mathbb{R}}^{2}$. Obviously, $f \notin G, W Y(G)=\langle I\rangle$ is finite but there exists no non-elementary group generated by $f$ and $g g^{-1}$ for $g \in G$.

Remark 3.2. Example 3.1 shows that Conjecture 1.4 does not hold.

Acknowledgements. The authors thank the referee very much for his (or her) valuable suggestions to this paper.

\section{References}

[1] Y. Jiang, On the discreteness of Möbius groups in all dimensions, Math. Proc. Camb. Phi. Soc. 136 (2004), no. 3, 547-555.

[2] T. Jørgensen, On discrete groups of Möbius transformations, Amer. J. Math. 98 (1976), $739-749$.

[3] no. 110, 209-211.

[4] L. Li and X. Wang, Discreteness criteria for Möbius groups acting on $\overline{\mathbb{R}}^{n} I I$, Bull. Aust. Math. Soc. 80 (2009), no. 2, 275-290.

[5] P. Tukia, Differentiability and rigidity of Möbius groups, Invent. Math. 82 (1985), no. $3,557-578$.

[6] P. Tukia and X. Wang, Discreteness of subgroups of $S L(2, \mathbb{C})$ containing elliptic elements, Math. Scand. 91 (2002), no. 2, 214-220.

[7] X. Wang, Dense subgroups of n-dimensional Möbius groups, Math. Z. 243 (2003), no. 4, 643-651.

[8] X. Wang, L. Li, and W. Cao, Discreteness criteria for Möbius groups acting on $\overline{\mathbb{R}}^{n}$, Israel J. Math. 150 (2005), 357-368.

[9] X. Wang and W. Yang, Discreteness criteria for subgroups in $S L(2, \mathbb{C})$, Math. Proc. Camb. Phi. Soc. 124 (1998), 51-55. 
$[10] \ldots$, Dense subgroups and discrete subgroups in $S L(2, \mathbb{C})$, Quart. J. Math. Oxford Ser. (2) 50 (1999), no. 200, 517-521.

[11] _ Generating systems of subgroups in $\operatorname{PSL}\left(2, \Gamma_{n}\right)$, Proc. Edinb. Math. Soc. (2) 45 (2002), no. 1, 49-58.

[12] _ Discreteness criteria of Möbius groups of high dimensions and convergence theorems of Kleinian groups, Adv. Math. 159 (2001), no. 1, 68-82.

[13] P. Waterman, Möbius transformations in several dimensions, Adv. Math. 101 (1993), no. $1,87-113$.

[14] (Berkeley, CA, 1986), 173-178, Math. Sci. Res. Inst. Publ., 11, Springer, New York, 1988.

LIULAN LI

Department of Mathematics and Computational Science

Hengyang Normal University

Hengyang, Hunan 421008, P. R. China

E-mail address: lanlimail2008@yahoo.com.cn

XI FU

Department of Mathematics

Hunan Normal University

Changsha, Hunan 410081, P. R. China 